\title{
Juvenile Probation Officers' Mental Health Decision Making
}

Gail A. Wasserman · Larkin S. McReynolds •

Andria L. Whited · Joseph M. Keating •

Hana Musabegovic · Yanling Huo

Published online: 23 September 2008

(C) Springer Science+Business Media, LLC 2008

Erratum to: Adm Policy Ment Health (2008) 35(5)

DOI 10.1007/s10488-008-0183-x

In the above mentioned article, Table 1 was incorrectly presented. Below please find the correct version of the table

The online version of the original article can be found under doi: 10.1007/s10488-008-0183-X.

G. A. Wasserman $(\bowtie) \cdot$ L. S. McReynolds .

A. L. Whited · J. M. Keating · H. Musabegovic · Y. Huo Division of Child and Adolescent Psychiatry, Center for the Promotion of Mental Health in Juvenile Justice, Columbia University/NY State Psychiatric Institute, 1051 Riverside Drive

(Unit 78), New York, NY 10032, USA

e-mail: wassermg@childpsych.columbia.edu 
Table 1 Characteristics of identified and not identified youth

\begin{tabular}{|c|c|c|c|c|c|}
\hline \multirow[t]{2}{*}{ Measure } & \multirow{2}{*}{$\begin{array}{l}\text { Total }(N=583) \\
\mathrm{n}(\%)\end{array}$} & \multicolumn{3}{|l|}{ Identified $(N=229)$} & \multirow{2}{*}{$\begin{array}{l}\text { Not identified } \\
(N=354) \\
\mathrm{n}(\%)\end{array}$} \\
\hline & & $\begin{array}{l}\text { Identified combined } \\
(n=229) \\
\mathrm{n}(\%)\end{array}$ & $\begin{array}{l}\text { Already identified } \\
(n=84) \\
\mathrm{n}(\%)\end{array}$ & $\begin{array}{l}\text { Newly identified } \\
(n=145) \\
\mathrm{n}(\%)\end{array}$ & \\
\hline Days chart open ${ }^{\mathrm{a}, * * \mathrm{~b}}$ & $72.4(88.9)$ & $106.9(106.8)$ & $108.4(117.9)$ & $106.0(100.2)$ & $50.0(66.1)$ \\
\hline \multicolumn{6}{|l|}{ Youth characteristics } \\
\hline Male & $423(73.4)$ & $176(77.2)$ & $64(77.1)$ & $112(77.2)$ & 247 (71.0) \\
\hline \multicolumn{6}{|l|}{ Race } \\
\hline White & $270(46.3)$ & 109 (47.6) & $47(55.9)$ & $62(42.8)$ & $161(45.5)$ \\
\hline African American & $247(42.4)$ & $95(41.5)$ & $28(33.3)$ & $67(46.2)$ & $152(42.9)$ \\
\hline Hispanic & $58(9.9)$ & $22(9.6)$ & $8(9.5)$ & $14(9.7)$ & $36(10.2)$ \\
\hline Other & $8(1.4)$ & $3(1.3)$ & $1(1.2)$ & $2(1.4)$ & $5(1.4)$ \\
\hline Age $(\text { years })^{\mathrm{a},{ }^{*} \mathrm{c}}$ & $14.0(1.5)$ & $14.1(1.4)$ & $13.8(1.7)$ & $14.3(1.2)$ & $14.0(1.6)$ \\
\hline Repeat offender ${ }^{* * * b}$ & $200(34.4)$ & $98(42.8)$ & $36(42.9)$ & $62(42.8)$ & $102(28.9)$ \\
\hline Age at first offense ${ }^{a, * * c}$ & $13.4(1.9)$ & $13.5(1.7)$ & $13.1(1.8)$ & $13.7(1.6)$ & $13.3(2.1)$ \\
\hline Violent offense ${ }^{* b}$ & $199(34.1)$ & $90(39.3)$ & $35(41.7)$ & $55(37.9)$ & $109(30.8)$ \\
\hline County MHPS ${ }^{* * * b}$ & $182(31.2)$ & $15(6.6)$ & $8(8.8)$ & $7(5.1)$ & $167(47.0)$ \\
\hline \multicolumn{6}{|l|}{ Probation Officer ${ }^{\mathrm{d}}$} \\
\hline Male & $238(46.6)$ & $84(43.1)$ & $26(36.1)$ & $58(47.1)$ & 154 (48.7) \\
\hline \multicolumn{6}{|l|}{$\operatorname{Race}^{* * * \mathrm{~b}, * \mathrm{c}}$} \\
\hline White & $421(82.4)$ & $182(93.3)$ & $71(98.6)$ & $111(90.2)$ & $239(75.6)$ \\
\hline Other & $90(17.6)$ & $13(6.7)$ & $1(1.4)$ & $12(9.8)$ & $77(24.4)$ \\
\hline $\mathrm{Age}^{\mathrm{a}}$ & $38.5(8.2)$ & $37.6(8.7)$ & $37.3(8.7)$ & $37.8(8.7)$ & $39.0(7.9)$ \\
\hline MH experience ${ }^{* * b}$ & $188(36.8)$ & $86(44.1)$ & $34(47.2)$ & $52(42.3)$ & $102(32.3)$ \\
\hline Years worked as PO & $8.6(8.1)$ & $7.9(8.4)$ & $6.6(7.0)$ & $8.7(9.0)$ & $9.0(7.90)$ \\
\hline Competency $^{\mathrm{a},{ }^{* * *} \mathrm{~b}, * * \mathrm{c}}$ & $3.9(0.5)$ & $4.0(0.5)$ & $3.9(0.5)$ & $4.1(0.5)$ & $3.8(0.5)$ \\
\hline Knowledge $^{\mathrm{a}, * * * \mathrm{~b},{ }^{*} \mathrm{c}}$ & $68.6(12.4)$ & $73.0(10.2)$ & $70.5(12.2)$ & $74.5(8.6)$ & $66.0(12.9)$ \\
\hline Self-efficacy ${ }^{\mathrm{a}, * * \mathrm{~b}, * *{ }^{\prime}}$ & $3.7(0.4)$ & $3.8(0.3)$ & $3.8(0.3)$ & $3.9(0.3)$ & $3.6(0.4)$ \\
\hline Opinions about services & $6.1(1.8)$ & $6.2(1.7)$ & $5.9(1.6)$ & $6.4(1.7)$ & $6.0(1.8)$ \\
\hline
\end{tabular}

Note: ${ }^{\text {a }}$ Values presented are means and standard deviations; ${ }^{\mathrm{b}}$ Identified and not identified youth significantly different; ${ }^{\mathrm{c}}$ Newly identified and already identified youth significantly different; ${ }^{\mathrm{d}}$ Marginal entries for POs do not reflect PO group characteristics, because those entries are weighted by the number of youths on a given PO's caseload

$* P<.05, * * P<.01, * * * P<.001$

MHPS $=$ Mental health professional shortage, as per US Health Resources and Services Administration; MH = Mental health; PO = Probation Officer 